\title{
La calidad de vida percibida por la familia que reside en la zona costera salvadoreña
}

\section{The perceived quality of life by families residing in the Salvadorean coastal zone}

\section{ISSN 2071-8748 \\ E-ISSN 2218-3345 \\ (c) (1) $\$(2)$ \\ BY NC SA}

URI: http://hdl.handle.net/11298/1141

DOI: https://doi.org/10.5377/entorno.v0i68.8461
Ana Sandra Aguilar de Mendoza' ORCID:0000-0003-0071-8795 José Rigoberto Vaquerano-Benavides ${ }^{2}$ Lizzie Náiera de Henríquez ${ }^{3}$ Larissa Hernández -Monterrosa ${ }^{3}$ Glenda Yamileth Trejo -Magaña ${ }^{4}$ Osmel Alberto Sánchez - Granados ${ }^{5}$ Elmer José René Hernández -Romero ${ }^{5}$ Edwin Osmil Coreas -Flores ${ }^{6}$

Diana Beatriz Moreno -Ventura ${ }^{6}$

Ramón Edgardo Marquina -Martínez ${ }^{6}$

Recibido: 6 de abril de 2019 Aprobado: 3 de julio de 2019

\section{Resumen}

El bienestar familiar adquiere importancia en la calidad de vida que una persona, familia o comunidad percibe. EI ODS 3, relacionado con gozar de bienestar, es el centro del desarrollo humano. Al explorar la percepción de calidad de vida, se utilizó la escala QLI-Sp en una muestra de 1.810 participantes que representan a familias que residen en los 20 municipios salvadoreños que tienen salida al océano Pacífico. Se aplicó la técnica de encuesta utilizando el software de QuestionPro para dispositivos móviles. La data se trabajó en el software de SSPS 19 para analizar la información recabada. Entre los resultados se encontró que la calidad de vida percibida

\section{Abstract}

A family's welfare acquires importance in terms of the perception of the quality of life that a person, a family, or a community perceives. The SDG 3, related to welfare, is the center of human development. In order to explore life quality in this study, the QLI-Sp scale was used in a sample of 1.810 participants who represent the families residing in the 20 Salvadorean municipalities on the shore of the Pacific Ocean. Surveys were conducted and QuestionPro was the software used for mobile devices. Data was analyzed with SPSS 19. Among the findings there can be mentioned that the quality of life, as perceived by the families living in the coastal zone of El Salvador, is

\footnotetext{
1 Universidad Tecnológica de El Salvador (Utec)

2 Universidad de Oriente (Univo)

3 Universidad Católica de El Salvador (Unicaes)

4 Universidad de Sonsonate (USO)

5 Universidad Gerardo Barrio (UGB), San Miguel

6 Universidad Gerardo Barrios (UGB), campus Usulután
} 
por las familias costeñas, en El Salvador, es deficiente porque no se superan, en más del $50 \%$ de sus miembros, las condiciones intrínsecas y extrínsecas que facilitan la percepción de una buena salud. Al no satisfacer sus necesidades materiales, las estructuras que sostienen la pobreza como los bajos salarios, las ocupaciones laborales, el tipo de actividad productiva, la falta de tenencia de la tierra, la práctica de actividades colectivas que integran a la familia, la inseguridad comunitaria, el poco acceso a la información y los obstáculos financieros para invertir en producción económica limitan la percepción de un mejor bienestar psicológico y emocional, reportando una deficiencia en el bienestar emocional, intrapersonal, en la plenitud personal y en el apoyo social/emocional. Las acciones de protección social para familias vulnerables presentan poca cobertura e inciden en la presencia de bienestar interpersonal.

\section{Palabras clave}

Costas del Océano Pacifico - El Salvador - calidad de vida. Litoral - El Salvador - Desarrollo humano. Costas del Océano Pacifico - El Salvador - Bienestar familiar. Costas del Océano Pacifico - El Salvador - aspectos psicológicos. Costas del Océano Pacifico - El Salvador - aspectos socioeconómicos.

\section{Introducción}

Este artículo es parte de un proyecto de investigación multicéntrico ejecutado por cinco universidades interesadas en el bienestar de las familias residentes en las áreas costeras salvadoreñas.

El avance en el Objetivos de Desarrollo Sostenible (ODS) de bienestar, requiere colocar a la salud en el centro del desarrollo humano. En cuanto a la salud de la población salvadoreña que reside en la zona costera, en el año 2005 la Organización Panamericana de la Salud (OPS) público el informe de país para Nicaragua, Guatemala y El Salvador basado en el instrumento IESM-OMS, que midió la salud mental. Para el 2004, los datos sobre la salud mental de la población salvadoreña indican que solo se invierte el $1 \%$ en este rubro y que el $99 \%$ se destina para el tratamiento psiquiátrico. Para este año se contabilizaron 49 establecimientos de salud que proporcionaron atención deficient since the intrinsic and extrinsic conditions that facilitate the perception of good health are not overcome for more than $50 \%$ of their members. By not meeting their material needs, the structures which sustain poverty such as low wages, people's occupations, the type of productive activity, the lack of land tenure, the practice of collective activities which help family integration, the lack of safety within the communities, the little access to information and the financial obstacles faced to be able to invest in the economic production limit the perception of a better emotional and psychological well-being, thus reporting a deficiency in the emotional and intrapersonal welfare of people, in their personal plenitude, and in the emotional/ social support. The actions taken for the social protection of vulnerable families demonstrate little coverage and affect the presence of interpersonal welfare.

\section{Keywords}

Pacific Ocean Coast - El Salvador - quality of life. Litoral - El Salvador - Human development. Pacific Ocean Coast - El Salvador - Family welfare. Pacific Ocean Coast - El Salvador - psychological aspects. Pacific Ocean Coast - El Salvador - socioeconomic aspects.

en salud mental de tipo ambulatorio. Los diagnósticos encontrados fueron principalmente relacionados con "síntomas neuróticos, estrés y somatomorfos en un $24 \%$ " (p. 52). No se reporta en el informe atenciones de seguimiento comunitario por no disponer de equipos móviles que atiendan en las comunidades. El informe reporta que no se encuentran tratamientos diurnos disponibles, ni residencias comunitarias para la atención de problemas psiquiátricos (OPS, 2006).

En la atención hospitalaria, para el 2004, la intervención tenía una predominancia del enfoque psicosocial con algún medicamento psicotrópico. En el informe de la OPS, también se destaca la proporción de un 25 \% de la población de sexo femenino que se atendió. Según las atenciones, un $21 \%$ de ellas fue clasificado con diagnóstico de "otras" (referido a alteraciones no diagnosticadas), "el $24 \%$ como trastornos neuróticos, el $14 \%$ como esquizofrenia y un $9 \%$ como trastornos de personalidad" (OPS, 2006, p. 57). 
Describiendo la situación de las familias salvadoreñas, surgen preguntas sobre la vida económica, psicosocial y su vinculación con el entorno y los recursos naturales con los que cuentan las familias que residen en la zona costeramarina de El Salvador, que son objeto de exploración en esta investigación como la siguiente pregunta: ¿La salud mental de los miembros de las familias puede identificarse a través de la calidad de vida y de la presencia de alteraciones en la salud de las familias que residen en estas zonas? Para responder este interrogante, se planteó el objetivo de identificar las alteraciones en la salud, así como la percepción de calidad de vida de las familias.

\section{Metodología}

Es un estudio cuantitativo, con un diseño descriptivo correlacional, en donde el diseño muestral está formado por una muestra con selección probabilística y una distribución por conglomerados de familias. La población es de 471.318 familias. Los participantes fueron 1.810 familias, representadas cada una por uno de sus miembros. Los criterios de inclusión de la familia estuvieron caracterizados por la apertura de la comunidad a través de directivas, líderes y lideresas comunitarios, la seguridad de la zona, el acompañamiento de las alcaldías a través de sus promotores sociales y el apoyo de los promotores de salud en algunas zonas. La muestra presenta una confiabilidad del $99 \%$ con un margen de error del $3 \%$. En la recolección de datos se incluyó: 5 puertos, 41 playas, 7 islas, 54 puntos que agrupan colonias, cantones y caseríos con salida al mar. Participaron 20 municipios, y entre las variables sociodemográficas se abordó la edad, el sexo, la residencia, el estado civil y el nivel educativo. La técnica utilizada fue la encuesta utilizando el software Question Pro, y en algunos casos de ubicación difícil o por dificultades en la señal de internet se aplicó la encuesta en papel.

En el instrumento elaborado por el grupo investigador, se incluyó una escala sobre índice de calidad de vida QLI-Sp, formada por 10 ítems, que miden el bienestar físico, el bienestar psicológico-emocional, el cuidado personal; el funcionamiento independiente, ocupacional e interpersonal, el apoyo emocional-social y el apoyo estatal y de servicio, la plenitud personal y espiritual y la percepción global de la calidad de vida. La escala es original de Mezzich, Cohen, Ruiperez, Yoon, Liu, adaptada por Lorente, Ibáñez, Moro, \& Ruipérez (2002) en una muestra española. El Alfa de Cronbach en la muestra final fue de .82 para 10 elementos. La medida de adecuación muestral KMO fue de .89 con un Chi cuadrado, en la prueba de esfericidad de Bartlett, de 4781.74, $\mathrm{p}<.00$.

\section{Resultados}

Para alcanzar el objetivo de identificar la percepción de calidad de vida de las familias residentes de la zona costero-marina de El Salvador, se encontró en el bienestar físico que más del $59 \%$ de los miembros de la familia reporta un bienestar físico deficiente, y solamente cerca de un $25 \%$ lo encuentra bueno. Esta deficiencia se debe a la poca capacidad para satisfacer sus necesidades de vivienda, alimentación, educación y salud (ver figura 1).

\section{Figura 1. Acceso a agua potable}

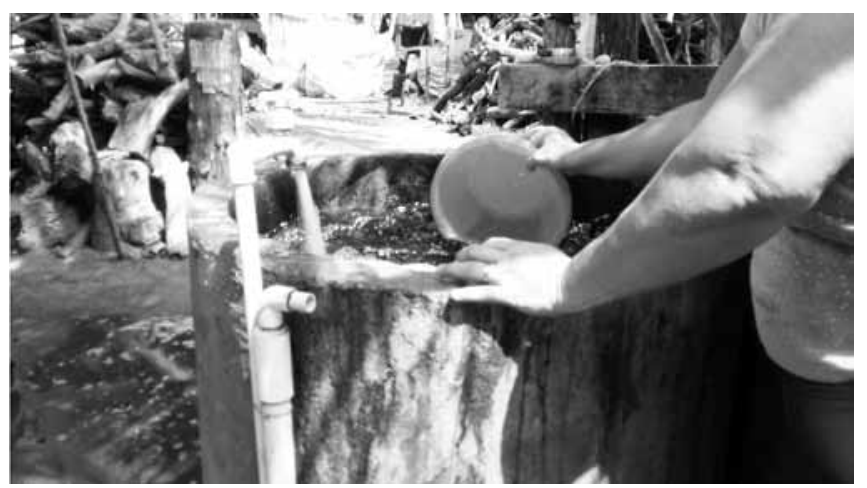

Fuente: Foto propiedad de la Utec, 2017.

Más del $40 \%$ de las familias también perciben un bienestar psicológico emocional deficiente. Utilizando pruebas $t$ de Studen, se encontraron diferencias estadísticas significativas de bienestar psicológico entre los que presentan síntomas de alteración de salud y los que no $\left(t(1733)=-8.35, \mathrm{M}_{(\mathrm{conAS})}=\right.$ $2.14, \mathrm{M}_{(\sin A S)}=1.79, p<.00$ ).

Al analizar el autocuidado y el funcionamiento independiente, más del $41 \%$ percibe que poseen un nivel bueno de bienestar en esta área, y un $37 \%$ percibe un bienestar deficiente (ver figura 26), el cual limita el realizar sus tareas cotidianas y la toma de decisiones para resolver problemas de forma independiente. En este estado de bienestar, las ocupaciones juegan un papel importante. Utilizando pruebas no paramétricas de Kruskal-Wallis, se muestra que las amas de casa reportan medias más altas de bienestar en autocuidado $y$ funcionamiento independiente $\left(K_{(7,1716)}=17.28, p=.02\right)$. En cuanto al funcionamiento ocupacional, más del $39 \%$ de las familias presentan una percepción de deficiencia. Su relación con las diversas ocupaciones de los miembros muestra diferencias estadísticas significativas según el empleo dominante en los miembros de la familia. Utilizando la prueba 
no paramétrica de Kruskal-Wallis, se encontraron diferencias estadísticas significativas de bienestar ocupacional entre las áreas de empleo $\left(K_{(933)}=5.34, p=, 02\right)$. El empleo en turismo reporta un buen funcionamiento ocupacional (14\%, $n=92)$, mientras que el empleo de pescador artesanal reporta un funcionamiento laboral deficiente $(31,9 \%, n=$ 199). La misma percepción de deficiencia en el bienestar ocupacional sucede en la comercialización de productos del mar y con los cuidadores de ranchos. Para las ocupaciones que no tienen una formalidad $(28,6 \%, n=456)$, y que realizan actividades diversas, más del $34 \%$ de esta muestra reportó un buen funcionamiento ocupacional.

Las relaciones interpersonales se valoraron dentro del bienestar interpersonal; y se encontró que más del $48 \%$ de las familias participantes se perciben con una buena relación con sus familiares, amigos y vecinos. Utilizando pruebas $C$ de contingencia, se encontró que las familias que practican actividades como reuniones familiares, deportes o paseos juntas presentan diferencias con las familias que no tienen estos factores protectores disponibles $\left(\mathrm{X} 2_{(2, \mathrm{n}=1723)}=29.40, p=\right.$ .00). Estas prácticas protectoras son reportadas en el $57 \%$ ( $n$ $=1031$ ) de las familias participantes. De ellas, el 30,5\% reportó una buena percepción de funcionamiento interpersonal. En el $42,5 \%$ de la muestra que no realiza prácticas protectoras como las mencionadas anteriormente, únicamente el 17,9\% reportó un buen funcionamiento personal.

El apoyo social emocional mostrado en la disposición de la familia para confiar y en la solicitud de pedir ayuda en caso de necesidad, el $47,7 \%(n=856)$ de los participantes reportó la percepción de un buen apoyo. Este apoyo se contrastó con los apoyos recibidos también de parte del Estado y de Ios familiares $\left(X 2_{(10, n=1785)}=22.61, p=.01\right)$. El 9,1\%(n=163) de las familias que reciben remesa reportaron una buena percepción de apoyo social emocional. El recibo de remesa es importante para percibir un bienestar, sin embargo, hay una proporción bastante significativa que no recibe apoyo económico de alguna fuente $(59,2 \%, n=1057)$; y perciben un buen apoyo social en más del $27 \%$, lo que indica que existen otros factores que inciden en la percepción del bienestar emocional que no fueron exploraos en esta investigación.

La percepción de bienestar comunitario se refiere a los apoyos comunitarios y servicios percibidos encontrados dentro de un vecindario seguro, los accesos a recursos financieros, el acceso a la información. El 41,2 \% $(n=740)$ percibe, en su entorno próximo, un buen apoyo comunitario; el $33,3 \%(n=598)$ un regular apoyo, y el $25,5 \%$, un déficit de apoyo comunitario.
La calidad de vida medida a través de la percepción de equilibrio entre el trabajo y el descanso con la práctica de actividades, como el disfrute sexual, el ocio, el sentimiento de equilibrio personal, la solidaridad y la dignidad, fue abordada mediante la presencia de plenitud personal. Más del $44 \%$ reportó una plenitud personal deficiente, que evidencia la existencia de un desequilibrio entre el descanso y el trabajo desempeñado. Una buena plenitud personal es percibida si se cuenta con factores protectores como salir a pasear, reunirse con sus familiares o practicar un deporte, ya que se generan espacios de convivencia $\left(U_{(1787)}=330456.50, Z=-5.99, R_{(s i}\right.$ factor) $=959.23, p<.00)$. En el disfrute de la sexualidad, no se exploró ampliamente debido a los comentarios surgidos entre las mujeres, que reportaron que la sexualidad es considerada solo para reproducción y no para el disfrute pleno. Es un tema que puede ampliarse posteriormente.

El sentimiento de fe, la religiosidad y la trascendencia se valoraron a través de la percepción de la plenitud espiritual. Entre los resultados, el $46 \%$ de las familias se perciben con una buena plenitud espiritual. Esta percepción varía según profesión de fe. Utilizando la prueba de Kruskal Wallis, se encontró que los que profesan la religión evangélica perciben una mayor espiritualidad $\left(H_{(6, n=1781)}=58.39, \mathrm{R}_{\text {(evangéicos) }}=\right.$ 958.76, $p=.00$ ) que las otras profesiones. La profesión de la fe católica ofrece una percepción de buena plenitud en segundo lugar $(R$ (católicos) $=958.75)$, y los que menos perciben una buena plenitud espiritual son los que no profesan alguna fe $\left(R_{\text {(ninguna) }}=730.40\right)$.

Los sentimientos de satisfacción y felicidad con la vida se midieron a través del índice de calidad de vida. El $42,6 \%$ de los participantes, reporto una buena percepción de su calidad de vida; sin embargo, el $36,8 \%$ percibe que su calidad de vida es deficiente. La satisfacción y felicidad con su vida no genera expectativas de desarrollo. Cuatro familias de cada 10 se perciben felices y satisfechas.

\section{Discusión de resultados}

\section{La calidad de vida en las familias residentes de las zonas costeras}

La calidad de vida incorpora la percepción de la persona sobre su felicidad, los indicadores sociales y la salud en general (Schwartzmann, 2003). Puede enfatizarse en la salud en general los funcionamientos relacionados consigo mismo y en sus entornos próximos, y en la salud mental (DuránArenas, Gallegos-Carrillo, Salinas-Escudero, \& Martínez- 
Salgado, 2004). La escala utilizada discrimina tanto la salud individual como colectiva: en la dimensión individual de la salud general se valoró el bienestar físico, el psicológico/ emocional y el autocuido tanto físico como mental. En la salud colectiva se abordó el bienestar interpersonal.

Los funcionamientos se valoraron en cuanto a las interacciones que correlacionan entre lo individual y lo colectivo: el funcionamiento independiente, el apoyo social emocional, el bienestar comunitario, el apoyo estatal y de servicio, la plenitud personal y espiritual. La salud mental se abordó desde la valoración de los estados de satisfacción y felicidad dentro de la percepción global de la calidad de vida.

El bienestar físico es una dimensión de la salud de las familias (Romera, 2003). En la muestra de familias, más del $59 \%$ no logran satisfacer sus necesidades materiales de vivienda, alimentación, educacion y salud. Esto implica que la estructura que sostiene la salud está debilitada, y esto aumenta la brecha de pobreza, las desigualdades y atrasa la posibilidad de cumplir con los ODS. Las limitaciones producidas por las estructuras son consecuentes con el bienestar psicológico/emocional reportado en esta investigación, donde más del $40 \%$ de la muestra reporto un deficiente bienestar psicológico, aumentado en aquellas familias donde sus miembros reportaron síntomas de alteraciones psicosomáticas, de salud mental y física. Estos datos muestran la integración de la persona en donde un déficit en el área física está correlacionado con un área mental y viceversa.

La dimensión del autocuido de la salud en general es importante para reducir la presencia de estrés, sobre todo el crónico, que es causa de desadaptación de la persona (González \& Landero, 2006). Este autocuido presenta indicadores de salud cuando se asocia con actividades ocupacionales o laborales que gozan de tiempos concretos de vida en donde ocurren diversos mecanismos para establecer logros de vida, reconocimientos de valía y valoración de la estima (Urquijo, 2014). En esta muestra de familias, las amas de casa se percibieron con un buen autocuido. Si bien es cierto las faenas son muy fuertes, estas ocurren sin presión para ser terminadas ese día. Las rutinas de aseo, cocina y lavado de ropa son básicas, por lo que los estresores valorados no reportan respuestas fisiológicas que alteren su salud (González \& Landero, 2006).

El bienestar percibido en cuanto al funcionamiento consigo mismo y con su entorno próximo (Lorente et al., 2002), muestra una relación con respecto a sus ocupaciones. Si bien es cierto las mujeres amas de casa se perciben con mejor autocuido, su funcionamiento ocupacional genera una percepción de deficiencia. Las ocupaciones laborales relacionadas con el turismo generan una percepción de un mayor bienestar, ya que la interacción con varias personas, la diversidad de actividades donde socializar y la obtención de mejores ganancias económicas producen mayor satisfacción. Las actividades pesqueras artesanales, por el contrario, generan una percepción deficiente de bienestar debido a los obstáculos o barreras de entrada ocurridas en la distribución y venta del producto pesquero (Oddone, Padilla, \& Antunes, 2014). La percepción es de trabajar mucho y recibir poco dinero. La venta directa de los productos del mar está relacionada con los consumidores, por lo tanto, la percepción cambiará en tanto sea en días feriados o fin de semana. Esta deficiente percepción de bienestar incide en que el comercio informal de diversos productos genere mejores percepciones de bienestar. Aunque es una ocupación no regulada puede lograr la circulación de dinero en efectivo a corto plazo (Aguilar, 2017). Esta salida les ayuda a mantener un flujo de dinero que satisface parcialmente sus necesidades materiales esenciales, convirtiéndose el comercio informal en una fuente de ingresos más aceptada; y que genera una percepción de bienestar diversa (ver figura 2).

\section{Figura 2. Venta de piñatas para comercializarlas en comunidades aledañas}

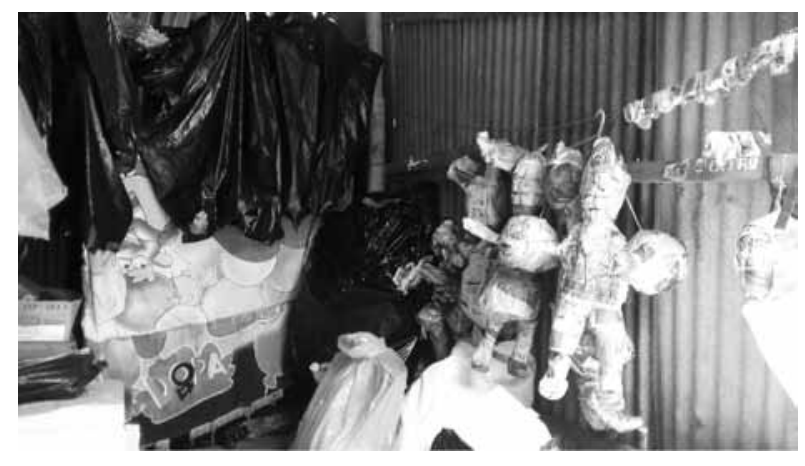

Fuente: Foto propiedad de la Utec, 2017.

La percepción de deficiencia en su bienestar también es importante para las familias que cuidan ranchos en la playa. Estas familias no pagan vivienda y tienen limitados los espacios para transitar. No surge el sentimiento de pertenencia, el trabajo que desempeñan es de tipo doméstico y de servicio a los dueños que llegan a descansar el fin de semana. El salario recibido por el jefe o jefa del hogar es más bajo que el recibido por los empleados domésticos urbanos, ya que se considera que los miembros de la familia 
son beneficiarios de los recursos de los propietarios, como el acceso gratuito a la energía eléctrica y el agua. Esta situación de no poder tener su propia vivienda genera la percepción de déficit.

El bienestar personal que las familias puedan percibir está relacionado también con sus funcionamientos. Las relaciones familiares, vecinales y comunitarias se ven aumentadas bajo condiciones de complejidad (Urquijo, 2014). Las familias extendidas que cuidan varios ranchos aledaños se perciben con mayor bienestar debido a que comparten experiencias de vida similares y se apoyan entre sí. El $48,4 \%$ de las familias participantes reportaron un buen funcionamiento interpersonal. Queda un segmento bastante grande del $31,2 \%(n=564)$, que expresa un déficit en su bienestar. El funcionamiento personal también está vinculado con factores protectores que son explicados a través de la convivencia con sus familiares como la reunión familiar, visita a otros familiares, salidas a practicar deporte 0 salidas a pasear en familia. En las familias que realizan estas prácticas protectoras $(n=1031)$, se reporta en más del $30 \%$ una buena percepción de funcionamiento interpersonal, mostrando que la convivencia familiar es importante para aumentar el bienestar de sus miembros.

Ese funcionamiento personal identificado tiene relación con la percepción de apoyo social/emocional encontrado en los miembros de las familias costeñas. En este estudio se encontró buena percepción de funcionamiento personal, tanto en familias que reciben remesa o apoyos estatales como en aquellas que no son beneficiarios de transferencias monetarias $\mathrm{u}$ otras fuentes. Las familias que reportaron remesas 0 ayudas del Estado $(47,7 \%, n=856)$ perciben un mejor bienestar interpersonal, mientras que en el grupo de familias que no tienen alguna fuente de apoyo económico solo el $17,9 \%$ reportó un buen funcionamiento. Las remesas y los programas de protección social en una familia se vuelven importantes dentro de la salud y el bienestar percibido. Para Urquijo (2014), la percepción de bienestar en la salud emocional es generada a partir de las demandas de factores externos, internos y estructurales que presionan ante la falta de apoyo. Es necesario ampliar la cobertura de los programas de protección social no contributiva para mejorar la percepción de bienestar en los miembros de la familia.

El bienestar social requiere de un apoyo comunitario que proporcione condiciones de seguridad e información. El apoyo comunitario facilita la organización comunitaria, la cual facilita el acceso a recursos financieros disponibles para una comunidad organizada. El $25 \%$ de las familias que residen en las áreas costeras reportaron un déficit de bienestar social. La comunidad por sí sola no valora la percepción de bienestar social al margen de las acciones del Estado (Aponte, 2012). En este estudio, los apoyos del Estado presentan poca cobertura en sus programas sociales, y son los programas sociales los que aumentan la posibilidad de convivir y tomar decisiones en cuanto a su seguridad y los mecanismos bajo los cuales aumentarán sus gestiones financieras. Solo 4 de 10 familias perciben un buen bienestar social. El incremento en la inseguridad, el poco acceso a la información y los obstáculos para invertir en proyectos productivos (Carcach, 2008) inciden en la percepción de bienestar de las familias residentes en la zona costera salvadoreña (ver figura 3).

\section{Figura 3. Granja familiar de huevos en la playa Toluca}

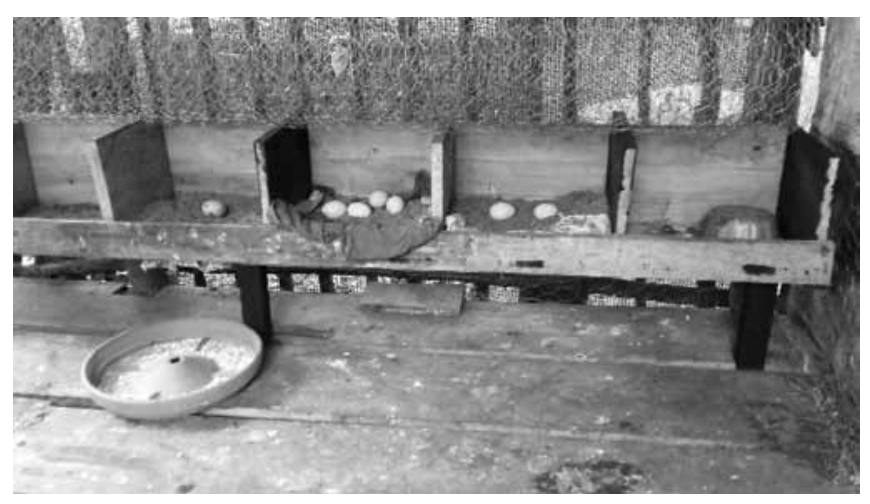

Fuente: Foto propiedad de la Utec, 2017.

Un funcionamiento importante para determinar bienestar es la presencia de plenitud en la vida de las familias. Esta plenitud se midió en dos áreas: personal y espiritual. La plenitud personal es favorecida cuando entre los miembros de la familia se realizan acciones o prácticas colectivas que se convierten en factores protectores. La interacción en actividades conjuntas como reunirse en familia, salir a pasear juntos 0 acudir a prácticas deportivas, como el fútbol, que predomina en la zona costera, produce una percepción de mayor plenitud personal. Dentro del grupo que no goza de factores protectores se percibe la plenitud personal como deficiente. Los programas sociales que conducen los gobiernos deben llegar hasta las comunidades costeras y no quedarse en las cabeceras departamentales.

En el goce de la plenitud espiritual, la religión tiene un papel importante en la calidad de vida. En el caso de las 
familias que residen en la zona costera, la profesión de fe evangélica provee condiciones para experimentar una mayor plenitud espiritual, mientras le sigue la profesión de fe católica. La presencia de las iglesias evangélicas dentro de varias comunidades supera la presencia de la Iglesia católica. Para estas familias, hay un contacto espiritual cercano a su residencia. El $46 \%$ reportó la percepción de una buena plenitud espiritual. Sin embargo, más del $36 \%$ que no profesa alguna religión declaró tener un déficit en su plenitud espiritual.

La deconstrucción de la calidad de vida a través de la percepción del bienestar en diferentes áreas de la vida humana muestra, en esta investigación, que la calidad de vida que presentan las familias que residen en el área costera no supera el $50 \%$ de las condiciones intrínsecas y extrínsecas que puedan facilitar la vivencia de una percepción de buena salud.

Tanto la presencia de alteraciones en la salud (físicas, psicológicas y sociales) de las familias, así como las condiciones de su entorno y los apoyos recibidos, inciden en la percepción de una salud buena, regular o deficitaria.

Al analizarse las diversas áreas, el patrón en las percepciones de bienestar identifica que globalmente solo 4 de cada 10 familias gozan de una calidad de vida favorable al desarrollo de sus miembros. Persisten las condiciones de déficit en las áreas económicas, sociales, psicológicas y culturales (ver figura 4).

\section{Figura 4. Percepción global de calidad de vida en las familias}

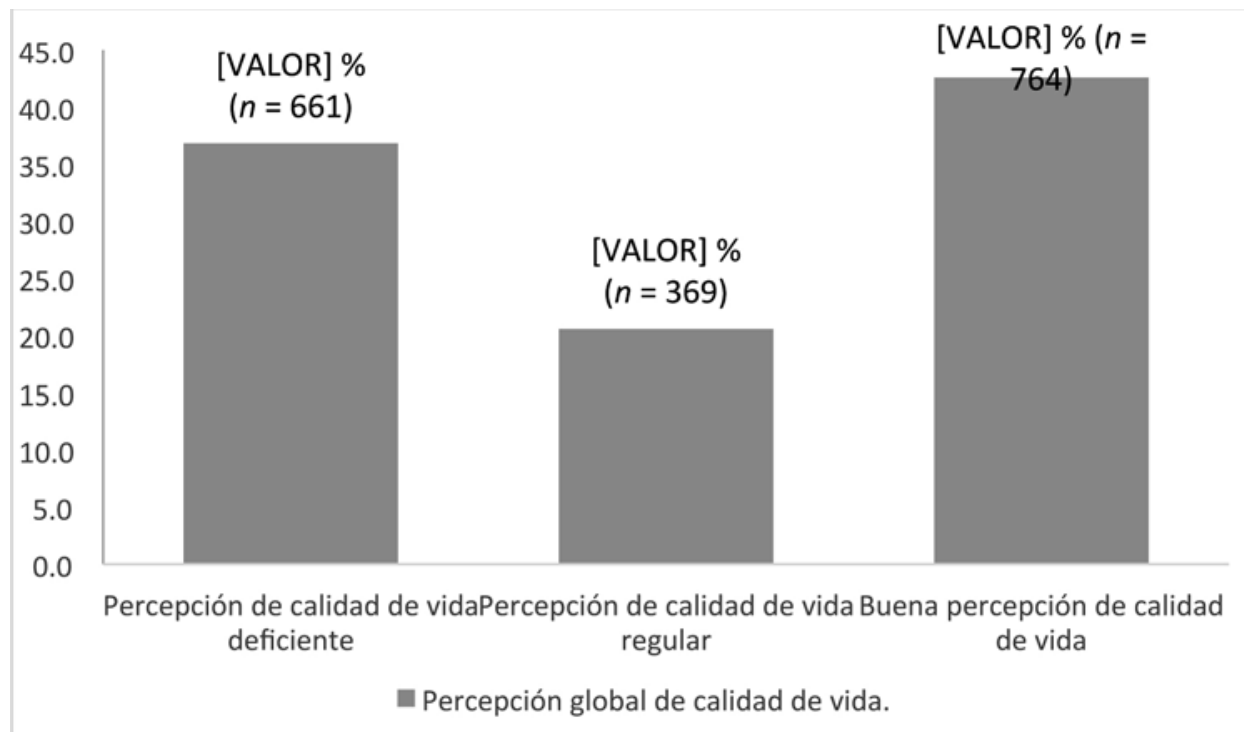

Fuente: Datos del estudio multicéntrico “El estado de las competencias de desarrollo socioeconómico y psicosocial de las familias en el área costera de El Salvador" (Aguilar et al., 2018).

Finalmente, la calidad de vida es una dimensión multifactorial, la cual puede intervenirse desde diversas áreas de actuación de la persona. En el caso de las familias residentes en la franja costero-marina salvadoreña, es necesario no solo apostar al desarrollo económico, sino ir paralelamente interviniendo con políticas públicas focalizadas dentro del marco del desarrollo local. 


\section{Referencias}

Aguilar de Mendoza,A.S. (2017).Elestadodelas competencias de desarrollo de la mujer en la zona de La Libertad [versión de Adobe Acrobat Reader]. Recuperado de https://www.utec.edu.sv/media/ publicaciones/flips/ coleccionInvestigaciones/2017/estado_desarrollo_ mujer_lalibertad/files/libroinvestigacion71.pdf

Aguilar de Mendoza, A. S., Vaquerano Benavides, J. R., Nájera de Henríquez, L., Hernández Monterrosa, L., Trejo Magaña, G. Y., Sánchez Granados, O. A.,... Marquina Martínez, R. E. (2018). El estado de las competencias de desarrollo socioeconómico y psicosocial de las familias en el área costera de El Salvador. Fase II. Estudio multicéntrico [versión de Adobe Acrobat Reader]. Recuperado de https://www.utec.edu.sv/ vips/uploads/investigaciones/investigacion82.pdf

Aponte Blank, C. (enero-julio, 2012). ¿Estado social o Estado de bienestar en América latina?. Revista venezolana de análisis de coyuntura. 18(1), 11-40.

Carcach, C. A. (2008). El Salvador. Mapa de violencia y su referencia histórica [versión de Adobe Acrobat Reader]. Recuperado de https://www.insumisos.com/Mapa\%20 de\%20violencia\%20en\%20El\%20Salvador.pdf

Durán-Arenas, L., Gallegos-Carrillo, K., Salinas-Escudero, G. y Martínez-Salgado, H. (julio-agosto, 2004). Hacia una base normativa mexicana en la medición de calidad de vida relacionada con la salud, mediante el Formato Corto 36. Salud Pública de México, 46(4), 306-315. Doi: https://doi.org/10.1590/S0036-36342004000400005

González Ramírez, M. T. y Landero Hernández, R. (junio, 2006). Síntomas psicosomáticos y teoría transaccional del estrés. Ansiedad y estrés, 12(1), 45-61.
Lorente, E., Ibañez, I., Moro, M. y Ruipérez, M. A. (marzoabril, 2002). Índice de calidad de vida: estandarización y características psicométricas en una muestra española. Psiquiatría y Salud Integral, 2(2), 45-50.

Odonne, N., Padilla Pérez, R. y Antunes, B. (2014). Metodología del proyecto CEPAL-GIZ para el diseño de estrategias de fortalecimiento de cadenas de valor. En R. Padilla Pérez (Ed.), Fortalecimiento de las cadenas de valor como instrumento de la política industrial. Metodología y experiencia de la CEPAL en Centroamérica (pp. 77111). Recuperado de https://repositorio.cepal.org/ handle/11362/36743

OPS/OMS (2006). IESM-OMS informe sobre los sistemas de salud mental en Nicaragua, El Salvador y Guatemala [versión de Adobe Acrobat Reader]. Recuperado de https://www.who.int/mental_health/ Nicaragua_ EISalvador_Guatemala_WHO-AIMS_Report2.pdf

Romera Iruela, M. J. (2003). Calidad de vida en el contexto familiar: dimensiones e implicaciones políticas. Intervención Psicosocial, 12(1), 47-63. Recuperado de https://www. redalyc.org/articulo.oa?id=179 818041002

Schwartzmann, L. (2003). Calidad de vida relacionada con la salud: aspectos conceptuales. Ciencia y enfermería, 9(2), 9-21. Recuperado de https://scielo.conicyt.cl/pdf/ cienf/v9n2/art02.pdf

Urquijo Angarita, M. J. (diciembre, 2014). La teoría de las capacidades en Amartya Sen. EDETANIA, (46), 63-80. Recuperado de https://dialnet.unirioja.es/descarga/ articulo/5010857.pdf 